\title{
Effect of dulaglutide on liver fat in patients with type 2 diabetes and NAFLD: randomised controlled trial (D-LIFT trial)
}

\author{
Mohammad S. Kuchay ${ }^{1} \cdot$ Sonal Krishan ${ }^{2} \cdot$ Sunil K. Mishra ${ }^{1} \cdot$ Narendra S. Choudhary $^{3} \cdot$ Manish K. Singh $^{4}$. \\ Jasjeet S. Wasir ${ }^{1} \cdot$ Parjeet Kaur $^{1} \cdot$ Harmandeep K. Gill $^{1} \cdot$ Tarannum Bano $^{1} \cdot$ Khalid J. Farooqui $^{1}$ • Ambrish Mithal ${ }^{1}$
}

Received: 25 May 2020 / Accepted: 22 July 2020 / Published online: 31 August 2020

(C) Springer-Verlag GmbH Germany, part of Springer Nature 2020

\begin{abstract}
Aims/hypothesis Liraglutide, a daily injectable glucagon-like peptide-1 receptor (GLP-1r) agonist, has been shown to reduce liver fat content (LFC) in humans. Data regarding the effect of dulaglutide, a once-weekly GLP-1r agonist, on human LFC are scarce. This study examined the effect of dulaglutide on LFC in individuals with type 2 diabetes and non-alcoholic fatty liver disease (NAFLD). Methods Effect of dulaglutide on liver fat (D-LIFT) was a 24 week, open-label, parallel-group, randomised controlled trial to determine the effect of dulaglutide on liver fat at a tertiary care centre in India. Adults $(n=64)$, who had type 2 diabetes and MRI-derived proton density fat fraction-assessed LFC of $\geq 6.0 \%$ at baseline, were randomly assigned to receive dulaglutide weekly for 24 weeks (add-on to usual care) or usual care, based on a predefined computer-generated number with a 1:1 allocation that was concealed using serially numbered, opaque, sealed envelopes. The primary endpoint was the difference of the change in LFC from 0 (baseline) to 24 weeks between groups. The secondary outcome measures included the difference of the change in pancreatic fat content (PFC), change in liver stiffness measurement (LSM in $\mathrm{kPa}$ ) measured by vibration-controlled transient elastography, and change in liver enzymes.

Results Eighty-eight patients were screened; 32 were randomly assigned to the dulaglutide group and 32 to the control group. Overall, 52 participants were included for per-protocol analysis: those who had MRI-PDFF data at baseline and week 24. Dulaglutide treatment resulted in a control-corrected absolute change in LFC of $-3.5 \%$ (95\% CI $-6.6,-0.4 ; p=0.025)$ and relative change of $-26.4 \%(-44.2$, $-8.6 ; p=0.004$ ), corresponding to a 2.6 -fold greater reduction. Dulaglutide-treated participants also showed a significant reduction in $\gamma-$ glutamyl transpeptidase (GGT) levels (mean between-group difference -13.1 U/1 [95\% CI -24.4, -1.8]; $p=0.025$ ) and non-significant reductions in aspartate aminotransferase (AST) $(-9.3 \mathrm{U} / 1[-19.5,1.0] ; p=0.075)$ and alanine aminotransferase (ALT) levels $(-13.1 \mathrm{U} / 1$ $[-24.4,2.5] ; p=0.10)$. Absolute changes in PFC $(-1.4 \%[-3.2,0.3] ; p=0.106)$ and $\operatorname{LSM}(-1.31 \mathrm{kPa}[-2.99,0.37] ; p=0.123)$ were not significant when comparing the two groups. There were no serious drug-related adverse events.

Conclusions/interpretation When included in the standard treatment for type 2 diabetes, dulaglutide significantly reduces LFC and improves GGT levels in participants with NAFLD. There were non-significant reductions in PFC, liver stiffness, serum AST and serum ALT levels. Dulaglutide could be considered for the early treatment of NAFLD in patients with type 2 diabetes.

Trial registration ClinicalTrials.gov NCT03590626

Funding The current study was supported by an investigator-initiated study grant from Medanta-The Medicity's departmental research fund and a grant from the Endocrine and Diabetes Foundation (EDF), India.
\end{abstract}

Keywords Controlled attenuation parameter $\cdot$ Dulaglutide $\cdot$ GLP-1 receptor agonists $\cdot$ Liver stiffness measurement $\cdot$ MRI-derived proton density fat fraction $\cdot$ MRI-PDFF $\cdot$ NAFLD $\cdot$ Non-alcoholic fatty liver disease $\cdot$ Non-alcoholic steatohepatitis $\cdot$ Pancreatic fat content

Electronic supplementary material The online version of this article (https://doi.org/10.1007/s00125-020-05265-7) contains peer-reviewed but unedited supplementary material, which is available to authorised users.

Mohammad S. Kuchay

drshafikuchay@gmail.com

Extended author information available on the last page of the article
Abbreviations

$\begin{array}{ll}\text { ALT } & \text { Alanine aminotransferase } \\ \text { AST } & \text { Aspartate aminotransferase } \\ \text { AWARD } & \begin{array}{l}\text { The Assessment of Weekly } \\ \text { Administration of Dulaglutide in Diabetes }\end{array} \\ \text { CAP } & \begin{array}{l}\text { Controlled attenuation parameter }\end{array}\end{array}$




\section{Research in context}

\section{What is already known about this subject?}

- A daily subcutaneous injectable glucagon-like peptide-1 receptor (GLP-1r) agonist, liraglutide, reduces liver fat and improves liver enzymes in individuals with NAFLD

- Data regarding the effect of dulaglutide, a once-weekly GLP-1r agonist, on NAFLD are scarce

What is the key question?

- Does dulaglutide treatment reduce liver fat and improve liver enzymes in individuals with coexisting type 2 diabetes and NAFLD?

What are the new findings?

- Dulaglutide-treated participants showed significant reduction in liver fat content and improvement in GGT levels

- Dulaglutide treatment also showed non-significant reductions in pancreatic fat content, liver stiffness, and serum AST and ALT levels

How might this impact on clinical practice in the foreseeable future?

- There is a huge unmet need for NAFLD treatment; dulaglutide could assist by reducing liver fat and improving liver enzymes in patients with coexisting type 2 diabetes and NAFLD

$\begin{array}{ll}\text { D-LIFT } & \text { Effect of dulaglutide on liver fat } \\ \text { DPP-4 } & \text { Dipeptidyl peptidase } 4 \\ \text { FPG } & \text { Fasting plasma glucose } \\ \text { GGT } & \gamma \text {-Glutamyl transpeptidase } \\ \text { GLP-1r } & \text { Glucagon-like peptide-1 receptor } \\ \text { LFC } & \text { Liver fat content } \\ \text { LSM } & \text { Liver stiffness measurement } \\ \text { MRI-PDFF } & \text { MRI-estimated proton density fat fraction } \\ \text { NAFLD } & \text { Non-alcoholic fatty liver disease } \\ \text { NASH } & \text { Non-alcoholic steatohepatitis } \\ \text { PFC } & \text { Pancreatic fat fraction } \\ \text { ROI } & \text { Region of interest } \\ \text { TSH } & \text { Thyrotropin } \\ \text { VCTE } & \text { Vibration-controlled transient elastography }\end{array}$

\section{Introduction}

Non-alcoholic fatty liver disease (NAFLD) frequently coexists with type 2 diabetes. The presence of type 2 diabetes in individuals with NAFLD is a risk factor for its progression to non-alcoholic steatohepatitis (NASH), a severe form of NAFLD that can further progress to liver fibrosis, cirrhosis and hepatocellular carcinoma [1]. Glucagon-like peptide-1 receptor (GLP-1r) agonists are glucose-lowering agents that have been demonstrated to reduce liver fat and improve liver histology in animal models of NASH [2-5]. GLP-1r agonists improve several pathogenetic processes involved in NAFLD. They have been shown to improve insulin sensitivity $[6,7]$, reduce fatty acid accumulation [8], inhibit NOD-like receptor 3 inflammasome activation [9], minimise endoplasmic reticulum stress [2], promote autophagy [2, 10], modulate gut microbiota [11] and modulate the enzymatic activity involved in fatty acid metabolism [12]. In human clinical studies, liraglutide reduces body weight, liver fat [12, 13] and improves liver histology [14] in individuals with NAFLD. However, two smaller studies did not show any benefit of liraglutide on liver fat $[15,16]$. Dulaglutide has been approved for the improvement of glycaemic control in adults with type 2 diabetes [17]. Dulaglutide is also approved for the reduction of major adverse cardiovascular events in adults with type 2 diabetes, with and without established CVD [18]. In a post hoc analysis of the Assessment of Weekly Administration of Dulaglutide in Diabetes (AWARD) programme, dulaglutide significantly reduced body weight and liver enzymes, especially in individuals with NAFLD [19]. Dulaglutide has shown better patient acceptability than other GLP-1r analogues [20]. The effect of dulaglutide on liver fat content (LFC) has not been evaluated so far. This prompted us to perform an RCT to elucidate the effect of dulaglutide on LFC in individuals with coexisting type 2 diabetes and NAFLD. Liver fat was measured by MRI-estimated proton density fat fraction (MRI-PDFF), which is a robust and accurate method of liver fat estimation [21-23]. Furthermore, pancreatic steatosis is a component of the metabolic syndrome [24] and is a risk factor for ductal adenocarcinoma of the pancreas [25]. One study did not find any reduction of pancreatic fat content (PFC) after 12 weeks of liraglutide therapy [26]. Therefore, we systematically evaluated the effect of dulaglutide on PFC assessed by MRI-PDFF. Additionally, we evaluated liver stiffness measurement (LSM) by vibration-controlled transient elastography (VCTE) as a 
surrogate marker for liver fibrosis, and measured liver enzyme (serum alanine aminotransferase [ALT], aspartate aminotransferase [AST] and $\gamma$-glutamyl transpeptidase [GGT]) levels.

\section{Methods}

Participants and setting This trial evaluating the effect of dulaglutide on liver fat (D-LIFT) was an investigator-initiated, open-label, RCT to examine the effect of dulaglutide on LFC when included in the standard treatment of type 2 diabetes vs standard treatment of type 2 diabetes in individuals with NAFLD. LFC was measured by MRI-PDFF. The study was designed and conducted in accordance with the CONSORT guidelines. The patient population for the trial was derived from Medanta-The Medicity Hospital endocrine outpatient clinic and who were primarily visiting for management of type 2 diabetes and other comorbidities. The study was conducted at Medanta-The Medicity Hospital, Gurugram, Haryana (which is a tertiary care centre in North India). Patients deemed eligible were screened for the trial. The clinical trial protocol was approved by the institutional review board and Ethics Committee (MICR-861/2018). Informed written consent was obtained from all the participants. The trial is registered with ClinicalTrials.gov (registration no. NCT03590626).

Eligibility criteria Patients were included if they met the following criteria: $>20$ years of age; had baseline MRIPDFF-assessed LFC of $\geq 6.0 \%$ [27]; had type 2 diabetes treated with metformin and/or dipeptidyl peptidase 4 (DPP-4) inhibitors and/or sulfonylurea and/or insulin, for whom treatment with dulaglutide was indicated due to poor diabetes control $\left(\mathrm{HbA}_{1 \mathrm{c}}>53 \mathrm{mmol} / \mathrm{mol}[>7.0 \%]\right)$; and provided written informed consent.

Patients were excluded if they met any of the following exclusion criteria: highly uncontrolled diabetes $\left(\mathrm{HbA}_{1 \mathrm{c}}\right.$ $>86 \mathrm{mmol} / \mathrm{mol}$ [>10.0\%]); alcohol consumption $>14$ units per week for women and $>21$ units per week for men; severe hepatic impairment of any cause (AST or ALT $>5$ times the upper limit of normal); evidence of other forms of liver disease, including hepatitis $\mathrm{B}$, hepatitis $\mathrm{C}$ and autoimmune hepatitis; use of drugs known to cause hepatic steatosis; treatment with glucose-lowering drugs that influence liver fat, including thiazolidinediones, $\alpha$-glucosidase inhibitors, sodium-glucose cotransporter 2 (SGLT2) inhibitors or any GLP-1r agonists during the previous 3 months; treatment with vitamin E during the previous 3 months; contraindications to dulaglutide use (history of acute or chronic pancreatitis or pancreatic cancer); and contraindications to MRI. See electronic supplementary material (ESM) Research design and methods.
Baseline assessment All the study participants underwent a baseline assessment before randomisation, including anthropometry, detailed medical history and physical examination. Alcohol assessment was documented using the AUDIT questionnaire [28]. All participants underwent blood tests, including glycaemic variables (fasting plasma glucose and $\mathrm{HbA}_{1 \mathrm{c}}$ ), liver function tests (total bilirubin, albumin, ALT, AST, GGT, alkaline phosphatase), kidney function tests (serum urea, creatinine, uric acid, spot urine protein/creatinine ratio), electrolytes (sodium, potassium), lipid profile (triacylglycerols, LDL, HDL), complete blood counts (haemoglobin, total leucocyte count, platelet count), hormones (thyrotropin [TSH]), hepatitis B surface antigen, anti-hepatitis C antibody and HIV I and II. At baseline, all participants underwent MRIPDFF examination as well as VCTE.

Randomisation and allocation concealment A research assistant (SR) randomised the participants into either the dulaglutide group or the control group based on a predefined computer-generated number with a 1:1 allocation that was concealed using serially numbered, opaque, sealed envelopes (prepared by a statistician). The participants were then sent to their respective consultants (MSK, SKM, KJF, JSW) in the endocrine department for adjustment of diabetic treatment (according to randomisation into dulaglutide or control groups). Treatment allocation was open-label. Investigators involved in imaging data analysis (i.e. radiology technician, radiologist, hepatologist) were blinded to participants' information and the allocation sequence. Although aware of the treatment group, the consultant endocrinologists were blinded to the imaging results until final data analysis. The drug used was dulaglutide (Trulicity; Eli Lilly, USA).

Study visits After careful assessment at the baseline visit, participants meeting all inclusion and exclusion criteria were randomised to receive dulaglutide $0.75 \mathrm{mg}$ by subcutaneous injection each week for 4 weeks, then $1.5 \mathrm{mg}$ weekly for 20 weeks plus standard treatment for type 2 diabetes. The control group only received standard care for type 2 diabetes. Before prescribing dulaglutide, history regarding current or past pancreatitis or unexplained abdominal pain, and personal or family history of current or previous thyroid malignancy were carefully documented. All participants were instructed about potential adverse drug reactions. Each participant was asked to document all the symptoms that they experienced during the study period, whether related to drug or not. In both groups, adjustment of diabetes treatment was carried out based on self-monitored blood glucose at weeks 4 and 8 by telephone consultation. We intended to maintain glycaemic equipoise between the two groups by adjusting glucoselowering medicines (metformin, DPP-4 inhibitors in the control group, sulfonylureas and/or insulin). This was intended to minimise the effect of correction of 
hyperglycaemia on liver fat in the two groups. Our prespecified targets for glycaemic variables for both the groups were according to the ADA guidelines 2018 [29]. All participants were instructed to restrict simple carbohydrates (avoid fruit juices and simple sugars, reduce rice preparations) and fat intake (reduce butter, ghee). All participants were advised to exercise (brisk walk) for at least $45 \mathrm{~min}$ a day for at least 5 days in a week.

Participants returned to the outpatient endocrine clinic for follow-up visits at weeks 12 and 24. All the participants enrolled in the study, at baseline and at week 12, received uniform lifestyle modification instructions from a consultant (MSK) in accordance with the standards of diabetes management [30]. See ESM Research design and methods for further details.

Outcomes The primary outcome measure was the difference of the change in LFC from week 0 (baseline) to week 24, as quantified by MRI-PDFF. Secondary outcome measures included the difference of the change in PFC from week 0 (baseline) to week 24, change in LSM (in $\mathrm{kPa}$ ) by VCTE from baseline, and changes in serum AST, ALT and GGT levels from baseline.

MRI-PDFF protocols MRI-PDFF is a non-invasive and quantitative biomarker that can accurately estimate LFC [21-23]. In this study, the mean \pm SD time interval from obtaining the baseline MRI-PDFF to initiating the study drug was $2 \pm$ 2 days. All MRI examinations were done by an experienced MRI technologist in the Medanta radiology department. All scans were analysed by a single trained radiologist investigator (SK) blinded to clinical data, treatment group allocation and the order (baseline or week 24) of each scan. We have previously validated the use of MRI-PDFF in the longitudinal assessment of liver fat in our institution [27]. To assess longitudinal changes in LFC, one colocalised region of interest (ROI; approximately $300-400 \mathrm{~mm}^{2}$ ) was placed in each of the nine liver segments (nine separate ROIs) on baseline and follow-up MRI examinations, avoiding blood vessels, bile ducts and artefacts. NAFLD was defined as MRI-PDFFassessed LFC $\geq 6.0 \%$ [27]. Similarly, one colocalised ROI was placed in each of the seven pancreatic regions (head, uncinate process, neck, proximal body, mid-body, distal body and tail) on baseline and follow-up MRI examinations. Non-alcoholic fatty pancreas disease (NAFPD) was defined as MRI-PDFF-assessed PFC $\geq 10.0 \%$ [31].

VCTE parameters LSM by VCTE (related to liver fibrosis) and controlled attenuation parameter (CAP; related to liver fat) were measured using Fibroscan 502 (Echosens, Paris, France) by a trained hepatologist (NSC) who was blinded to the drug allocation, clinical data and MRI-PDFF data. Following a fast of at least $3 \mathrm{~h}$, all participants underwent
Fibroscan (with M or XL probe) examination at baseline and again at week 24. Measurements were considered valid when there were at least ten accurate shots, the success rate was at least $60 \%$, and the IQR was less than $30 \%$ of the median values of the CAP and LSM. The final CAP and LSM values were recorded as the medians of all measurements.

Biochemical measurements at follow-up History of prescribed medications and their adverse reactions were noted and anthropometry, physical examination results and biochemical measurements were recorded for each participant at week 24 . Venous blood samples were taken in the morning after participants had fasted overnight for $12 \mathrm{~h}$ and the samples were analysed on the same day at the centre laboratory of the hospital for the following variables: fasting plasma glucose (FPG); $\mathrm{HbA}_{1 \mathrm{c}}$; liver function test (total bilirubin, albumin, AST, ALT, GGT, alkaline phosphatase); kidney function test (urea, creatinine, uric acid, urine protein/creatinine ratio), lipid profile (triacylglycerols, HDL, LDL); electrolytes (sodium, potassium, calcium); complete blood counts (haemoglobin, total leucocyte count, platelet count); and TSH.

Sample size calculation We assumed that a $5.0 \%$ absolute difference in LFC between dulaglutide and control groups would be the minimally appreciable and clinically relevant difference. Based on the results of previous similar clinical studies involving ezetimibe, sitagliptin and empagliflozin $[27,32,33]$, we expected the dulaglutide group to have an absolute liver fat reduction of $>5.0 \%$ compared with baseline and the control group to have $<2.0 \%$ reduction in liver fat compared with baseline, and a dropout rate of $<10 \%$. With these assumptions, the sample size per group worked out as $\geq 22$ to achieve a power of at least $90 \%$ with a $\beta$ of 0.05 . Therefore, we planned to randomise 64 participants (32 in each group) to ensure adequate study power even with dropouts.

Statistical procedures Categorical data are presented as $n(\%)$. Continuous descriptive data were examined for normality and normally distributed data are presented as mean $\pm \mathrm{SD}$. Skewed data are presented as median (IQR). To compare the two groups, the $\chi^{2}$ test or Fisher's exact test were used for categorical variables, and the independent sample Student's $t$ test or Wilcoxon-Mann-Whitney $U$ test were used to compare differences between continuous variables. The Pearson correlation coefficients $(r)$ were determined by linear regression analysis. Additional analyses of primary and secondary outcomes within treatment groups were performed by using two-tailed independent sample $t$ tests, paired $t$ tests or nonparametric tests, when indicated. Univariate logistic regression was performed to screen for potential covariates associated with liver fat improvement. Covariates with $p<0.01$ were entered into the multivariable model using the forward 
condition method. Multivariate linear regression analysis was conducted to reveal the estimate effect of dulaglutide on liver fat. Statistical analyses were performed by a biostatistician (MKS). All the statistical analyses were performed using the SAS System version 9.4 (SAS Institute, Cary, NC, USA).

\section{Results}

Description of study population Between 10 November 2018 and 20 August 2019, 64 individuals with type 2 diabetes and NAFLD were randomised to receive either dulaglutide in addition to standard treatment for type 2 diabetes (dulaglutide group) or standard treatment for type 2 diabetes (control group). A total of 88 individuals were assessed for eligibility (Fig. 1). Overall, 52 participants were included for perprotocol analysis: those who had MRI-PDFF data at baseline and week 24. In the dulaglutide arm, three participants developed adverse drug reactions related to dulaglutide treatment and discontinued the study, two were lost to follow-up, and 27 participants completed the study. In the control arm, two participants discontinued the study due to protocol violation, five were lost to follow-up, and 25 completed the study. Thirty per cent of the study population was women, and all were of Indian origin. The two groups had comparable baseline characteristics (Table 1). The estimated compliance rate for dulaglutide treatment was $>95 \%$, as estimated by direct interviewing on follow-up visits. Following randomisation, DPP-4 inhibitors were discontinued in all participants who were assigned to the dulaglutide group, as both medicines are not recommended simultaneously. Table 1 shows the medications that our study participants were taking at enrolment (baseline).

Effect of dulaglutide on LFC LFC was significantly reduced in the dulaglutide group compared with the control group. Dulaglutide-treated participants showed a greater reduction in LFC compared with control participants at week 24 $(-32.1 \%$ vs $-5.7 \%$, respectively; mean difference $-26.4 \%$ [95\% CI $-44.2,-8.6] ; p=0.004)$. Similar changes were seen in absolute LFC (Table 2, Fig. 2). Compared with baseline, there was a significant difference in end-of-treatment LFC in the dulaglutide group (from $17.9 \%$ to $12.0 \%, p=<0.0001$ ) and an insignificant change in the control group (from $17.1 \%$ to $14.8 \%, p=0.058$ ) (see ESM Table 1 ). Seven patients $(26 \%)$ in the dulaglutide group achieved LFC below $6.0 \%$ on MRI-PDFF compared with two (8\%) in the control group after 24 weeks (ESM Table 2).

Effect of dulaglutide on PFC At baseline, $43.7 \%$ in the control group and $33.3 \%$ in the dulaglutide group had pancreatic steatosis. Dulaglutide-treated participants showed an insignificant reduction in PFC. The mean between-group difference in absolute PFC was $-1.4 \%, p=0.106$ (Table 2). Compared with baseline, there was a significant difference in the end-oftreatment PFC in the dulaglutide group (from $9.3 \%$ to $7.2 \%$, $p=0.006$ ) and an insignificant change in the control group (from $8.8 \%$ to $8.1 \%, p=0.198$ ) (ESM Table 3 ).

Effect of dulaglutide on transient elastography parameters Dulaglutide-treated participants demonstrated a nonsignificant reduction in LSM compared with the control group (mean difference $-1.31 \mathrm{kPa}$ [95\% CI -2.99, 0.37]) (Table 2). Compared with baseline, there was a significant reduction in the end-of-treatment LSM in the dulaglutide group (from $10.8 \mathrm{kPa}$ to $9.3 \mathrm{kPa}, p=0.016$ ) (ESM Table 4).

Similarly, dulaglutide treatment resulted in a nonsignificant reduction in CAP compared with the control group (mean difference $-21.0 \mathrm{~dB} / \mathrm{m}[95 \% \mathrm{CI}-43.1,1.2]$ ) (ESM Tables 4 and 5).

Effect of dulaglutide on body weight Dulaglutide-treated participants showed a significant reduction in body weight at 24 weeks when compared with the control group. The mean difference in absolute body weight change between the two groups was $-2.3 \mathrm{~kg}(95 \% \mathrm{CI}-4.1,-0.6)(p=0.01)$ (Table 3$)$. Compared with baseline, both the groups showed significant weight loss (from $85.8 \mathrm{~kg}$ to $81.5 \mathrm{~kg}$ in the dulaglutide group, $p<0.0001$; from $83.7 \mathrm{~kg}$ to $81.7 \mathrm{~kg}$ in the control group, $p<0.0001)$. Similar significant changes were seen in BMI (Table 3). More participants in the dulaglutide group than in the control group showed weight loss of $\geq 5 \%$ ( $24.0 \%$ control vs $55.6 \%$ dulaglutide) at the end of study period; $36.0 \%$ of the participants in the control group experienced weight gain compared with $7.4 \%$ in the dulaglutide group (ESM Table 6).

Effect of dulaglutide on serum AST, ALT and GGT levels Comparison of the dulaglutide and control groups showed that there was a significant difference for change in serum GGT $(-13.1 \mathrm{U} / 1, p=0.025)$ and non-significant differences in AST $(-9.3 \mathrm{U} / 1, p=0.075)$ and ALT levels $(-13.1 \mathrm{U} / 1, p=0.10)$ (Table 3). Compared with baseline, there was significant difference in end-of-treatment AST levels in both the groups (from $50 \mathrm{U} / 1$ to $33 \mathrm{U} / 1$ in the dulaglutide group, $p<0.0001$; from $46 \mathrm{U} / 1$ to $39 \mathrm{U} / 1$ in the control group, $p<0.0001$ ) (ESM Table 7). Similar significant reductions were seen in the endof-treatment ALT levels in both the groups.

Maintenance of glycaemic equipoise in the two groups There was a significant decrease in FPG and $\mathrm{HbA}_{1 \mathrm{c}}$ in both the dulaglutide group (FPG fell from $9.9 \mathrm{mmol} / \mathrm{l}$ to $6.3 \mathrm{mmol} / \mathrm{l}$, $p<0.0001 ; \mathrm{HbA}_{1 \mathrm{c}}$ fell from $69 \mathrm{mmol} / \mathrm{mol}$ to $51 \mathrm{mmol} / \mathrm{mol}$ [8.5\% to $6.8 \%], p<0.0001)$ and the control group (FPG fell from $9.5 \mathrm{mmol} / \mathrm{l}$ to $6.9 \mathrm{mmol} / \mathrm{l}, p<0.0001 ; \mathrm{HbA}_{1 \mathrm{c}}$ fell from $68 \mathrm{mmol} / \mathrm{mol}$ to $54 \mathrm{mmol} / \mathrm{mol}$ [8.4\% to $7.1 \%], p<0.0001$ ) (ESM Table 7). However, there were no significant 
Table 1 Baseline characteristics of study population

Characteristic $\quad$ Control $(n=32) \quad$ Dulaglutide $(n=32)$

Demographics

Age, years
Male sex
Comorbidities
Type 2 diabetes $^{\text {Hypertension }}{ }^{\mathrm{a}}$
Hyperlipidaemia $^{\mathrm{b}}$
Hypothyroidism $^{\mathrm{CVD}}$

Concomitant drug use

Glucose-lowering agents

Metformin

DPP-4 inhibitor

Sulfonylurea

Insulin

BP-lowering agent

Lipid-lowering agent

Antiplatelet agent

Proton pump inhibitor

Oral colecalciferol

Smoking history

Current smoker

Ex-smoker

Never smoked

Metabolic variables

Weight, $\mathrm{kg}$

BMI, $\mathrm{kg} / \mathrm{m}^{2}$

Duration of diabetes, years

Systolic BP, mm/Hg

Diastolic BP, $\mathrm{mm} / \mathrm{Hg}$

Fasting glucose, $\mathrm{mmol} / \mathrm{l}$

$\mathrm{HbA}_{1 \mathrm{c}}, \mathrm{mmol} / \mathrm{mol}$

$\mathrm{HbA}_{1 \mathrm{c}}, \%$

Kidney function tests

Serum urea, mmol/1

Serum creatinine, $\mu \mathrm{mol} / 1$

Serum uric acid, mmol/1

Urine protein/creatinine ratio

Electrolytes

Serum sodium, $\mathrm{mmol} / \mathrm{l}$

Serum potassium, $\mathrm{mmol} / \mathrm{l}$

$140 \pm 2.2$

$4.5 \pm 0.3$

Serum total calcium, $\mathrm{mmol} / \mathrm{l}$

$2.4 \pm 0.1$

Liver function tests

Total bilirubin, $\mu \mathrm{mol} / 1$

Serum albumin, $\mathrm{g} / 1$

AST, U/1

ALT, U/1

GGT, U/1

$13.4 \pm 6.6$

$44.4 \pm 2.4$

$10.7 \pm 4.3$

$46.1 \pm 21.1$

$42.9 \pm 3.7$

$68.1 \pm 30.8$

$49.9 \pm 22.7$

Alkaline phosphatase, U/1
$60.9 \pm 38.7$

$105.3 \pm 42.1$
Table 1 (continued)

\begin{tabular}{lll}
\hline Characteristic & Control $(n=32)$ & Dulaglutide $(n=32)$ \\
\hline Lipids & & \\
$\quad$ Triacylglycerols, $\mathrm{mmol} / 1$ & $2.2 \pm 0.9$ & $2.5 \pm 1.1$ \\
$\quad$ HDL-cholesterol, $\mathrm{mmol} / 1$ & $1.0 \pm 0.2$ & $1.0 \pm 0.2$ \\
$\quad$ LDL-cholesterol, $\mathrm{mmol} / 1$ & $2.7 \pm 0.7$ & $2.8 \pm 0.8$ \\
Blood counts & & \\
$\quad$ Haemoglobin, $\mathrm{g} / \mathrm{l}$ & $142 \pm 14.8$ & $137 \pm 18.1$ \\
$\quad$ Total leucocytes, $\times 10^{3} / \mu \mathrm{l}$ & $7.5 \pm 1.7$ & $8.0 \pm 2.3$ \\
$\quad$ Platelets, $\times 10^{3} / \mu \mathrm{l}$ & $186.2 \pm 51.9$ & $189.9 \pm 78.9$ \\
Hormones & & \\
$\quad$ THS, $\mu \mathrm{U} / \mathrm{ml}$ & $2.7 \pm 1.4$ & $3.5 \pm 1.9$ \\
\hline
\end{tabular}

Data are $n(\%)$ or mean \pm SD except where indicated

None of the baseline characteristics were significantly different between groups

${ }^{\text {a }}$ Hypertension was defined as treatment with a BP-lowering drug or having a detected $\mathrm{BP} \geq 140 / 90 \mathrm{mmHg}$ at screening for the study

${ }^{\mathrm{b}}$ Hyperlipidaemia was defined as treatment with a lipid-lowering drug or having a detected high lipid level during screening for the study

differences in $\mathrm{FPG}$ or $\mathrm{HbA}_{1 \mathrm{c}}$ between the two groups at the end of treatment (mean difference $-0.9 \mathrm{mmol} / 1[95 \% \mathrm{CI}-2.7$, $0.8]$ for FPG, $p=0.296 ;-3.7 \mathrm{mmol} / \mathrm{mol}[-0.3 \%]$ for $\mathrm{HbA}_{1 \mathrm{c}}$, $p=0.218$ ) (Table 3 ). We intended to maintain glycaemic equipoise between the two groups, by adjusting other glucoselowering medicines, so that the changes in glycaemic variables between the two group were similar.

Clinical and biological variables and their correlations with reduction in LFC The correlations between the reduction in LFC and modifications in clinical variables following 24 weeks of dulaglutide treatment are shown in ESM Table 8. The reductions in LFC correlated significantly with the reductions in body weight (ESM Fig. 1), $\mathrm{HbA}_{1 \mathrm{c}}$ (ESM Fig. 2), serum AST levels (ESM Fig. 3) and serum triacylglycerol levels (ESM Fig. 4). The correlation between the reduction in LFC and the reduction in body weight was highly significant $(r=0.495, p<0.01)$.

We also analysed the association between the change in LFC and the change in clinical variables in participants treated with dulaglutide. A multivariate analysis was performed by introducing variations into the model that correlated with the reduction in LFC with a $p$ value of $<0.001$. We also analysed the effect of various potential confounding factors such as age and sex on LFC reduction. The decrease in LFC was independently associated with the decrease in body weight $(p=$ $0.011)$, decrease in $\mathrm{HbA}_{1 \mathrm{c}}(p=0.039)$ and decrease in triacylglycerol levels $(p=0.008)$ (ESM Table 9). The results of the multivariate analysis were similar when treatment with glucose-lowering drugs (i.e. metformin, sulfonylurea, insulin) was introduced into the model. 
Fig. 1 Flow chart for the study cohort. UGI, upper gastrointestinal; ULN, upper limit of normal

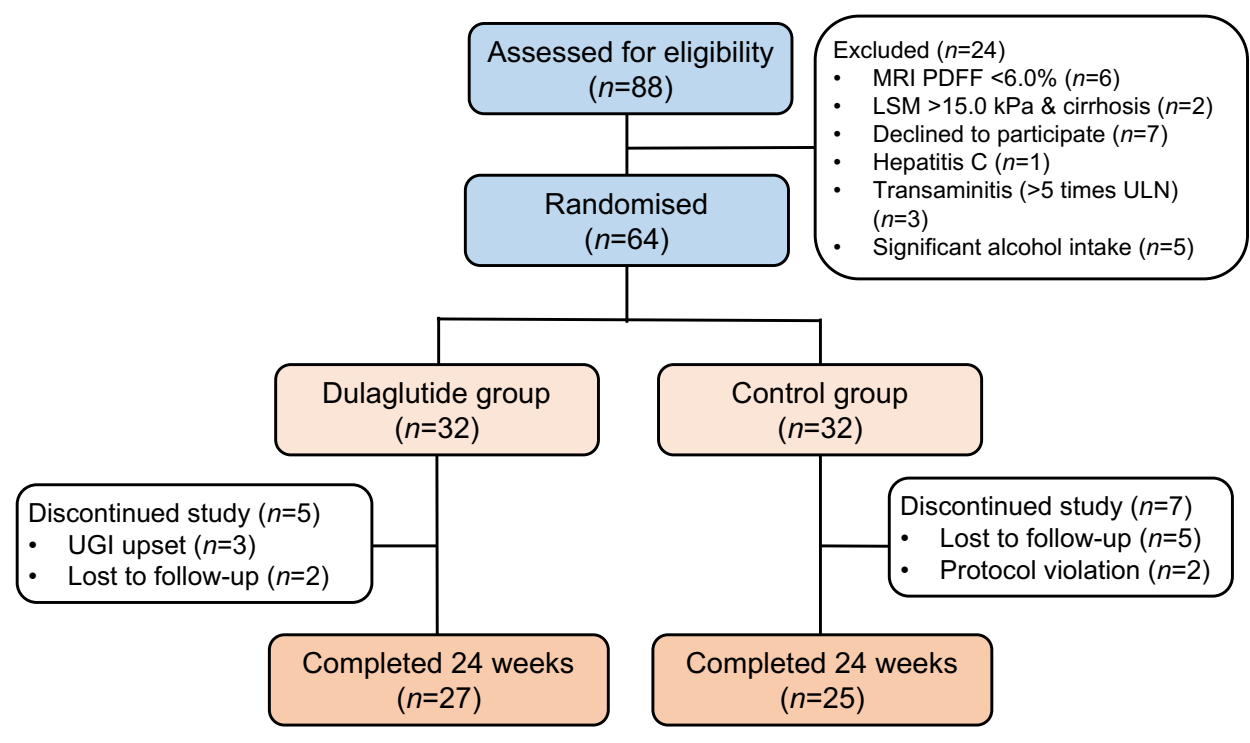

In our study, there was a significant correlation between dulaglutide-induced decrease in LFC and baseline LFC ( $r=$ $0.482 ; p=0.011$ ) (ESM Fig. 5). On further analysis, when baseline LFC was included in the statistical model, we found a significant and independent association between decrease in LFC and baseline LFC $(p<0.0001)$, reduction in body weight $(p<0.0001)$ and reduction in triacylglycerol levels $(p=0.019)$ (ESM Table 10).

Safety profile Three participants in the dulaglutide arm developed upper gastrointestinal upset in the initial weeks of treatment. Despite antacid and proton pump inhibitor therapy, these individuals preferred not to continue the therapy. Two further participants in the dulaglutide arm developed mild upper gastrointestinal upset but this resolved after antacid and proton pump inhibitor treatment. One participant developed transient diarrhoea after first two doses of dulaglutide. No-one developed abdominal pain in the dulaglutide treatment arm. Two participants in the control arm developed upper gastrointestinal symptoms, probably due to metformin up-titration, but they subsequently resolved without any change in medication. Two participants in the control arm dropped out of the study because of work scheduling and another three for personal reasons. The dropouts from the control arm were not associated with any drug adverse events (Fig. 1).

\section{Discussion}

In this RCT, which was performed in a usual care setting among patients with type 2 diabetes and NAFLD, the results showed that when included in the treatment for type 2 diabetes, dulaglutide significantly reduced LFC and improved serum GGT levels. The mean between-group difference in absolute and relative change in LFC was $-3.5 \%(p=0.025)$ and $-26.4 \%(p=0.004)$, respectively.

To the best of our knowledge, there are no studies to date that have evaluated the effect of dulaglutide on LFC. However, a few clinical studies have evaluated the effect of liraglutide on LFC. In the Liraglutide Efficacy and Action in NASH (LEAN) trial, liraglutide reduced LFC and improved liver histology in patients with biopsy-proven NASH [14]. Following 48 weeks of treatment with liraglutide, resolution of NASH without worsening fibrosis (primary outcome) occurred in 39\% patients compared with $9.0 \%$ patients in the placebo group. In another study using magnetic resonance spectroscopy for LFC estimation, liraglutide treatment for 6 months resulted in a significant relative LFC reduction of $31 \%$ [13]. We used MRI-PDFF for liver fat estimation and found that dulaglutide had an effect on LFC similar to that seen with liraglutide. Two small studies did not show reduction of LFC following liraglutide treatment $[15,16]$. Both these studies were of short-duration (12 weeks), so probably this is the reason that these studies failed to show any reduction in LFC. All the studies with duration of at least 24 weeks have shown reduction of LFC after liraglutide therapy [13, 14].

In our study, dulaglutide-treated participants showed a significant reduction in body weight compared with the control participants $(-2.3 \mathrm{~kg})$ over the 24 weeks. Body weight reduction is a class effect of all GLP-1r agonists [13, 14]. Body weight reduction was a significant factor driving the decrease in LFC following dulaglutide treatment in our study. In a study of individuals with histologically proven NASH, reduction in body weight through lifestyle changes was found to result in significant improvements in the histological features of NASH [34]. The authors found that these improvements were independently associated with the degree of body weight loss. In another clinical study, body weight reduction 
Table 2 Baseline and change (absolute and relative) in LFC, PFC and LSM following 24 weeks of treatment (per-protocol analysis)

\begin{tabular}{cllll}
\hline & Control $(n=25)$ & $\begin{array}{l}\text { Dulaglutide } \\
(n=27)\end{array}$ & $\begin{array}{l}\text { Between-group } \\
\text { difference, \% (95\% CI) }\end{array}$ & $p$ value \\
\hline MRI-PDFF assessment & & & & - \\
Baseline LFC, \% (mean \pm SD) & $17.1 \pm 7.7$ & $17.9 \pm 7.2$ & - & - \\
Week 24 LFC, \% (mean \pm SD) & $14.8 \pm 6.5$ & $12.0 \pm 6.6$ & - & $0.025^{*}$ \\
Absolute LFC change, \% (SE) & $-2.3(1.2)$ & $-5.8 \%(1.0)$ & $-3.5(-6.6,-0.4)$ & $0.004^{*}$ \\
Relative LFC change, \% (SE) & $-5.7(7.9)$ & $-32.1(4.3)$ & $-26.4(-44.2,-8.6)$ & - \\
Baseline PFC, \% (mean \pm SD) & $8.8 \pm 6.7$ & $9.3 \pm 7.0$ & - & - \\
Week 24 PFC, \% (mean \pm SD) & $8.1 \pm 5.1$ & $7.2 \pm 4.5$ & - & 0.106 \\
Absolute PFC, \% change (SE) & $-0.7(0.5)$ & $-2.1(0.7)$ & $-1.4(-3.2,0.3)$ & 0.192 \\
Relative PFC change, \% (SE) & $-0.2(5.2)$ & $-11.4(6.6)$ & $-11.2(-28.3,5.8)$ & - \\
LSM by VCTE & & & & - \\
Baseline LSM, kPa (mean \pm SD) & $9.0 \pm 4.3$ & $10.8 \pm 5.1$ & - & 0.123 \\
Week 24 LSM, kPa (mean \pm SD) & $8.9 \pm 4.9$ & $9.3 \pm 4.3$ & - & 0.124 \\
Absolute LSM change, kPa (SE) & $-0.12(0.63)$ & $-1.43(0.56)$ & $-1.31(-2.99,0.37)$ & $-11.2(-25.5,3.5)$ \\
Relative LSM change, kPa (SE) & $2.04(25.7)$ & $-9.1(25.7)$ & - & \\
\hline
\end{tabular}

*Statistically significant $p$ values after bypass surgery resulted in a marked improvement of liver steatosis, inflammation and fibrosis [35]. Multivariate analyses of our study indicated that the LFC reduction in participants receiving dulaglutide was independently and significantly associated with the decrease in body weight. Similar findings were noted in individuals receiving liraglutide [13].

In our study, the difference between the two groups observed for change in serum GGT levels was significant $(p=0.025)$. Although there were significant reductions in serum AST and ALT levels in the dulaglutide arm, the

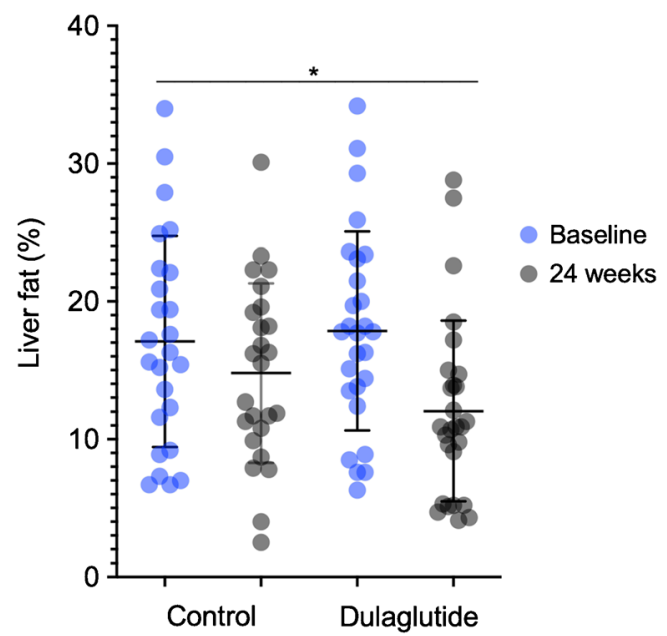

Fig. 2 Baseline and post-treatment absolute changes in MRI-PDFFassessed LFC in dulaglutide and control groups. $* p=0.025$ for the mean change from baseline (dulaglutide vs control). Data are presented as mean \pm SD. Coloured circles represent the LFC of each patient at baseline and grey circles represent the LFC of each patient at week 24. Between-group differences (change from baseline) were tested for significance using ANCOVA between-group differences did not reach statistical significance. In the AWARD programme analysing data of 1499 participants, dulaglutide significantly reduced AST, ALT and GGT levels compared with placebo, especially in patients with NAFLD [19]. Significant reductions in serum ALT $(p=$ $0.021)$ and GGT $(p=0.017)$ levels following liraglutide treatment were observed in another study [13].

In our study, we estimated liver stiffness by transient elastography, which is a surrogate marker for liver fibrosis. It must be noted that it is the hepatic fibrosis stage that predicts hepatic and cardiovascular morbidity and mortality in patients with NAFLD [36]. We did not note significant reduction in LSM following dulaglutide treatment for 24 weeks; however, neither was there any worsening of liver stiffness in the dulaglutide group. Long-term biopsy studies are needed to elucidate whether dulaglutide reduces liver fibrosis.

Pancreatic steatosis is a component of the metabolic syndrome [24, 37]. Obesity is a risk factor for pancreatic cancer [38]. At baseline, $42 \%$ of the participants in our study population had fatty pancreas. The dulaglutide-treated participants showed a significant decrease in PFC $(p=0.006)$ and a non-significant decrease was observed in the control group $(p=0.198)$. However, the mean between-group difference in absolute PFC change was not significant $(p=0.106)$ (Table 2). Similar non-reduction in PFC was also seen after liraglutide treatment [26].

In our study, we maintained glycaemic equipoise between the two groups, so that the effect of blood-glucose reduction on liver fat was similar in the two groups. Our study showed a significant correlation between decrease in LFC and decrease in $\mathrm{HbA}_{1 \mathrm{c}}(r=0.512 ; p=0.006)$ following dulaglutide treatment (ESM Fig. 2). It has been established that de novo lipogenesis in the liver is stimulated by carbohydrate-responsive 
Table 3 Changes in clinical and biochemical variables following 24 weeks of treatment (per-protocol analysis)

\begin{tabular}{lcccc}
\hline Variable & $\begin{array}{l}\text { Control (SE) } \\
(n=25)\end{array}$ & $\begin{array}{l}\text { Dulaglutide (SE) } \\
(n=27)\end{array}$ & $\begin{array}{l}\text { Mean between- } \\
\text { group } \\
\text { difference }(95 \% \mathrm{CI})\end{array}$ & $\begin{array}{l}p \\
\text { value }\end{array}$ \\
\hline Body weight, kg & $-2.0(0.7)$ & $-4.3(0.5)$ & $-2.3(-4.1,-0.6)$ & $0.01^{*}$ \\
BMI, kg/m & $-0.68(0.3)$ & $-1.5(0.2)$ & $-0.8(-1.43,-0.19)$ & $0.01^{*}$ \\
FPG, mmol/1 & $-2.6(2.8)$ & $-3.6(3.5)$ & $-0.9(-2.7,0.8)$ & 0.296 \\
$\mathrm{HbA}_{1 \mathrm{c}}, \mathrm{mmol} / \mathrm{mol}$ & $-13.7(2.4)$ & $-17.4(1.8)$ & $-3.7(-9.6,2.3)$ & 0.218 \\
$\mathrm{HbA}_{1 \mathrm{c}}, \%$ & $-1.3(0.2)$ & $-1.6(0.2)$ & $-0.3(-0.9,0.2)$ & 0.222 \\
Total bilirubin, $\mu \mathrm{mol} / \mathrm{l}$ & $-1.8(3.4)$ & $-0.9(4.3)$ & $-0.9(-1.3,3.1)$ & 0.407 \\
AST, U/1 & $-7.3(3.8)$ & $-16.6(3.4)$ & $-9.3(-19.5,1.0)$ & 0.075 \\
ALT, U/1 & $-13.9(5.0)$ & $-26.4(5.5)$ & $-13.1(-24.4,2.5)$ & 0.1 \\
GGT, U/1 & $-6.3(4.6)$ & $-19.4(3.4)$ & $-4.6(-15.5,6.3)$ & 0.401 \\
Alkaline phosphatase, U/1 & $-5.6(3.4)$ & $-10.2(4.2)$ & $-0.3(-0.8,0.2)$ & 0.187 \\
Triacylglycerols, mmol/1 & $-0.2(1.0)$ & $-0.5(0.7)$ & $-0.1(-0.1,0.2)$ & 0.391 \\
HDL-cholesterol, mmol/1 & $-0.0(0.2)$ & $-0.1(0.2)$ & $0.1(-0.3,0.6)$ & 0.592 \\
LDL-cholesterol, mmol/1 & $-0.4(0.9)$ & $-0.2(0.6)$ & $0.3(-0.3,0.9)$ & 0.271 \\
Serum urea, mmol/1 & $0.1(1.0)$ & $0.2(1.1)$ & $-5.5(-11.2,0.3)$ & 0.06 \\
Serum creatinine, mmol/1 & $3.6(11.2)$ & $-1.9(9.3)$ & $0(-0.1,0)$ & 0.11 \\
Serum uric acid, mmol/1 & $0(0.1)$ & $0(0.1)$ & $-0.15(-0.26,-0.04)$ & $0.01^{*}$ \\
Urine protein/creatinine & $-0.0(0.01)$ & $-0.15(0.05)$ & & $0.025^{*}$ \\
$\quad$ ratio & & & & \\
\hline
\end{tabular}

*Statistically significant $p$ values element-binding protein (CREBP) and sterol-regulatory element-binding protein 1c (SREBP-1c) transcription factors, which are in turn stimulated by hyperglycaemia and hyperinsulinaemia, respectively $[39,40]$. In our study, improvement of blood glucose levels in both the groups may have diminished stimulation of these transcription factors, thus reducing de novo lipogenesis in the liver. As improvement in blood glucose also occurred in the control group undergoing usual care, this may explain partial nonsignificant reduction in LFC in the control group.

Our study population was receiving other medications for diabetes and/or concurrent ailments (Table 1 and ESM Table 11). Metformin is reported to have modest or no effect on LFC reduction [41]. All our participants, in both the groups (100\% in each group), were receiving treatment with metformin. Forty per cent (10 out of 25 ) in the control group were taking DPP-4 inhibitors. DPP-4 inhibitors have been shown to have minimal effect on liver fat in patients with type 2 diabetes [33]. Sixteen per cent of participants in the control group and $11.1 \%$ in the dulaglutide group were also undergoing levothyroxine therapy. However, all the participants had stable TSH levels in the normal reference range for at least 6 months prior to recruitment into the study. An equal number of participants in either group were on proton pump inhibitors ( $8 \%$ in the control group and $7.4 \%$ in the dulaglutide group). Therefore, the effect of proton pump inhibitors on liver fat reduction (if any) in our study would have been negligible. More participants in the control group (56\%) were taking sulfonylureas than in the dulaglutide group (33\%) at the end of the study. However, on multivariate analysis, when glucose-lowering drugs (e.g. sulfonylureas) were introduced into the model as confounding factors, the results remained unchanged.

Strengths and limitations The strength of this study lies in its use of an RCT design to evaluate the efficacy of dulaglutide for reducing LFC. This study was conducted in a real-world scenario where patients were getting standard treatment for type 2 diabetes and other comorbidities. The second strength of this study is the use of MRI-PDFF for liver fat quantification. MRI-PDFF is a precise and accurate imaging biomarker.

We acknowledge some limitations of our study. First, we did not use placebo in the control group, as this study was conducted in a real-world scenario. Rather, we provided standard care for type 2 diabetes and other comorbidities. Second, MRI-PDFF only provides information regarding changes in LFC and does not provide any information regarding inflammation, hepatocyte ballooning degeneration and fibrosis. However, we used VCTE (Fibroscan) for assessing liver stiffness (a surrogate marker for liver fibrosis). Third, our participants were using other medications for type 2 diabetes and comorbidities. Although, individuals using medications that had known effects on liver fat were excluded from the study, minor interactions of co-prescribed 
medications on liver fat cannot be ruled out. Fourth, we could not document the extent of energy restriction and physical activity achieved by our study population. We gave uniform lifestyle instructions to all the participants according to ADA guidelines. Fifth, the study has a small sample size and larger studies are warranted to confirm these findings.

Conclusion When included in the standard treatment for type 2 diabetes, dulaglutide significantly reduced LFC and improved serum GGT levels. The results also indicated non-significant improvements in serum AST and ALT levels. Our study suggests that dulaglutide may be a useful agent for reducing liver fat accumulation, which often coexists with type 2 diabetes. Paired biopsy-based studies are needed to see whether liver fat reduction following dulaglutide treatment leads to improvement in NASH and fibrosis scores.

Acknowledgements We want to thank S. Rao (research coordinator, Division of Endocrinology and Metabolism, Medanta-The Medicity Hospital, Gurugram) for his help in the conduct of the study. We also want to thank the Endocrine and Diabetes foundation (EDF), India for providing a grant for this study.

Data availability The dataset for the current study is available on reasonable request by contacting MSK.

Funding The current study was supported by an investigator-initiated study grant to MSK from Medanta-The Medicity's departmental research fund and a grant from the Endocrine and Diabetes Foundation (EDF), India. The funding agency did not have any role in the design and conduct of the study, collection, management, analysis or interpretation of the data, or in the preparation, review or approval of the manuscript.

Authors' relationships and activities MSK has received speaker honoraria from Novartis, Sanofi, Wockhardt Limited, AstraZeneca and Novo Nordisk. SKM has received speaker honoraria from Sanofi, Novartis, AstraZeneca, Novo Nordisk, Boehringer Ingelheim, Lupin Limited and Abbott India Limited. JSW has received speaker honoraria from Boehringer Ingelheim, Novartis, Sanofi, Abbott India Limited, Wockhardt Limited, AstraZeneca and Novo Nordisk. AM has received speaker honoraria from Johnson and Johnson, Amgen, Sanofi, Novartis, AstraZeneca, Novo Nordisk, Boehringer Ingelheim, Lupin Limited and Abbott India Limited. All other authors declare that there are no relationships or activities that might bias, or be perceived to bias, their work.

Contribution statement MSK was responsible for the study concept and design, data collection, interpretation of data and drafting of the manuscript. SK was responsible for MRI analysis and interpretation. SKM and KJF were responsible for patient referral and data collection. NSC was responsible for Fibroscan performance and data collection. MKS was responsible for statistical analysis and data collection. JSW was responsible for patient referral and analysis of data. PK, HKG and TB were responsible for data collection. AM was responsible for the study concept, patient referral, study supervision and obtaining funding. All authors had access to study data, critically reviewed the manuscript and approved the final version. MSK is the guarantor of this work and, as such, had full access to all the data in the study and takes responsibility for the integrity of the data and the accuracy of the data analysis.

\section{References}

1. Angulo P (2002) Nonalcoholic fatty liver disease. N Engl J Med 346(16):1221-1231. https://doi.org/10.1056/nejmra011775

2. Sharma S, Mells JE, Fu PP, Saxena NK, Anania FA (2011) GLP-1 analogs reduce hepatocyte steatosis and improve survival by enhancing the unfolded protein response and promoting macroautophagy. PLoS One 6(9):e25269. https://doi.org/10.1371/ journal.pone.0025269

3. Ding X, Saxena NK, Lin S, Gupta NA, Anania FA (2006) Exendin4, a glucagon-like protein-1 (GLP-1) receptor agonist, reverses hepatic steatosis in ob/ob mice. Hepatology 43(1):173-181. https://doi.org/10.1002/hep.21006

4. Lee J, Hong SW, Chae SW et al (2012) Exendin-4 improves steatohepatitis by increasing Sirt1 expression in high-fat dietinduced obese C57BL/6J mice. PLoS One 7(2):e31394. https:// doi.org/10.1371/journal.pone.0031394

5. Trevaskis JL, Griffin PS, Wittmer C et al (2012) Glucagon-like peptide-1 receptor agonism improves metabolic, biochemical, and histopathological indices of nonalcoholic steatohepatitis in mice. Am J Physiol Gastrointest Liver Physiol 302(8):G762-G772. https://doi.org/10.1152/ajpgi.00476.2011

6. Mells JE, Fu PP, Sharma S et al (2012) GLP-1 analog, liraglutide, ameliorates hepatic steatosis and cardiac hypertrophy in C57BL/6J mice fed a Western diet. Am J Physiol Gastrointest Liver Physiol 302(2):G225-G235. https://doi.org/10.1152/ajpgi.00274.2011

7. Zhou JY, Poudel A, Welchko R et al (2019) Liraglutide improves insulin sensitivity in high fat diet induced diabetic mice through multiple pathways. Eur J Pharmacol 861:172594. https://doi.org/ 10.1016/j.ejphar.2019.172594

8. Bouchi R, Nakano Y, Fukuda T et al (2017) Reduction of visceral fat by liraglutide is associated with ameliorations of hepatic steatosis, albuminuria, and micro-inflammation in type 2 diabetic patients with insulin treatment: a randomized control trial. Endocr J 64(3):269-281. https://doi.org/10.1507/endocrj.ej16-0449

9. Zhu W, Feng PP, He K, Li SW, Gong JP (2018) Liraglutide protects non-alcoholic fatty liver disease via inhibiting NLRP3 inflammasome activation in a mouse model induced by high-fat diet. Biochem Biophys Res Commun 505(2):523-529. https://doi. org/10.1016/j.bbrc.2018.09.134

10. He Q, Sha S, Sun L, Zhang J, Dong M (2016) GLP-1 analogue improves hepatic lipid accumulation by inducing autophagy via AMPK/mTOR pathway. Biochem Biophys Res Commun 476(4): 196-203. https://doi.org/10.1016/j.bbrc.2016.05.086

11. Moreira GV, Azevedo FF, Ribeiro LM et al (2018) Liraglutide modulates gut microbiota and reduces NAFLD in obese mice. $\mathrm{J}$ Nutr Biochem 62:143-154. https://doi.org/10.1016/j.jnutbio.2018. 07.009

12. Kawaguchi T, Itou M, Taniguchi E, Sata M (2014) Exendin-4, a glucagon-like peptide-1 receptor agonist, modulates hepatic fatty acid composition and $\Delta$-5-desaturase index in a murine model of non-alcoholic steatohepatitis. Int J Mol Med 34(3):782-787. https:// doi.org/10.3892/ijmm.2014.1826

13. Petit JM, Cercueil JP, Loffroy R et al (2017) Effect of liraglutide therapy on liver fat content in patients with inadequately controlled type 2 diabetes: the Lira-NAFLD study. J Clin Endocrinol Metab 102(2):407-415. https://doi.org/10.1210/jc.2016-2775

14. Armstrong MJ, Gaunt P, Aithal GP et al (2016) Liraglutide safety and efficacy in patients with non-alcoholic steatohepatitis (LEAN): a multicentre, double-blind, randomised, placebo-controlled phase 2 study. Lancet 387(10019):679-690. https://doi.org/10.1016/ s0140-6736(15)00803-x

15. Smits MM, Tonneijck L, Muskiet MHA et al (2016) Twelve week liraglutide or sitagliptin does not affect hepatic fat in type 2 
diabetes: a randomised placebo-controlled trial. Diabetologia 59(12):2588-2593. https://doi.org/10.1007/s00125-016-4100-7

16. Tang A, Rabasa-Lhoret R, Castel H et al (2015) Effects of insulin glargine and liraglutide therapy on liver fat as measured by magnetic resonance in patients with type 2 diabetes: a randomized trial. Diabetes Care 38(7):1339-1346. https://doi.org/10.2337/dc142548

17. Fala L (2015) Trulicity (dulaglutide): a new GLP-1 receptor agonist once-weekly subcutaneous injection approved for the treatment of patients with type 2 diabetes. Am Health Drug Benefits 8:131-134

18. Gerstein HC, Colhoun HM, Dagenais GR et al (2019) Dulaglutide and cardiovascular outcomes in type 2 diabetes (REWIND): a double-blind, randomised placebo-controlled trial. Lancet 394(10193):121-130. https://doi.org/10.1016/s0140-6736(19) 31149-3

19. Cusi K, Sattar N, García-Pérez LE et al (2018) Dulaglutide decreases plasma aminotransferases in people with type 2 diabetes in a pattern consistent with liver fat reduction: a post hoc analysis of the AWARD programme. Diabet Med 35(10):1434-1439. https:// doi.org/10.1111/dme.13697

20. Li Z, Zhang Y, Quan X et al (2016) Efficacy and acceptability of glycemic control of glucagon-like peptide-1 receptor agonists among type 2 diabetes: a systematic review and network meta-analysis. PLoS One 11(5):e0154206. https://doi.org/10.1371/journal. pone.0154206

21. Noureddin M, Lam J, Peterson MR et al (2013) Utility of magnetic resonance imaging versus histology for quantifying changes in liver fat in nonalcoholic fatty liver disease trials. Hepatology 58(6): 1930-1940. https://doi.org/10.1002/hep.26455

22. Patel NS, Peterson MR, Brenner DA, Heba E, Sirlin C, Loomba R (2013) Association between novel MRI-estimated pancreatic fat and liver histology determined steatosis and fibrosis in nonalcoholic fatty liver disease. Aliment Pharmacol Ther 37(6):630 639. https://doi.org/10.1111/apt.12237

23. Reeder SB (2013) Emerging quantitative magnetic resonance imaging biomarkers of hepatic steatosis. Hepatology 58(6):1877-1880. https://doi.org/10.1002/hep.26543

24. Lee JS, Kim SH, Jun DW et al (2009) Clinical implications of fatty pancreas: correlations between fatty pancreas and metabolic syndrome. World J Gastroenterol 15(15):1869-1875. https://doi. org/10.3748/wjg.15.1869

25. Hori M, Takahashi M, Hiraoka N et al (2014) Association of pancreatic fatty infiltration with pancreatic ductal adenocarcinoma. Clin Transl Gastroenterol 5(3):e53. https://doi.org/10.1038/ctg. 2014.5

26. Smits MM, Tonneijck L, Muskiet MHA et al (2017) Pancreatic effects of liraglutide or sitagliptin in overweight patients with type 2 diabetes: a 12-week randomized, placebo-controlled trial. Diabetes Care 40(3):301-308. https://doi.org/10.2337/dc16-0836

27. Kuchay MS, Krishan S, Mishra SK et al (2018) Effect of empagliflozin on liver fat in patients with type 2 diabetes and nonalcoholic fatty liver disease: a randomized controlled trial (ELIFT trial). Diabetes Care 41(8):1801-1808. https://doi.org/10. 2337/dc18-0165

28. Johnson JA, Lee A, Vinson D, Seale JP (2013) Use of AUDITbased measures to identify unhealthy alcohol use and alcohol dependence in primary care: a validation study. Alcohol Clin Exp
Res 37(Suppl 1):E253-E259. https://doi.org/10.1111/j.1530-0277. 2012.01898.x

29. American Diabetes Association (2018) Glycemic targets: standards of medical care in diabetes-2018. Diabetes Care 41(Suppl 1):S55S64. https://doi.org/10.2337/dc18-s006

30. American Diabetes Association (2018) Lifestyle management: standards of medical care in diabetes-2018. Diabetes Care 41(Suppl 1):S38-S50. https://doi.org/10.2337/dc18-s004

31. Wong VW, Wong GL, Yeung DK et al (2014) Fatty pancreas, insulin resistance, and $\beta$-cell function: a population study using fat-water magnetic resonance imaging. Am J Gastroenterol 109(4):589-597. https://doi.org/10.1038/ajg.2014.1

32. Loomba R, Sirlin CB, Ang B et al (2015) Ezetimibe for the treatment of nonalcoholic steatohepatitis: assessment by novel magnetic resonance imaging and magnetic resonance elastography in a randomized trial (MOZART trial). Hepatology 61(4):1239-1250. https://doi.org/10.1002/hep.27647

33. Cui J, Philo L, Nguyen P et al (2016) Sitagliptin vs. placebo for non-alcoholic fatty liver disease: a randomized controlled trial. J Hepatol 65(2):369-376. https://doi.org/10.1016/j.jhep.2016.04.021

34. Vilar-Gomez E, Martinez-Perez Y, Calzadilla-Bertot L et al (2015) Weight loss through lifestyle modification significantly reduces features of nonalcoholic steatohepatitis. Gastroenterology 149(2): 367-378. https://doi.org/10.1053/j.gastro.2015.04.005

35. Mattar SG, Velcu LM, Rabinovitz M et al (2005) Surgicallyinduced weight loss significantly improves nonalcoholic fatty liver disease and the metabolic syndrome. Ann Surg 242(4):610-617. https://doi.org/10.1097/01.sla.0000179652.07502.3f

36. Ekstedt M, Hagström H, Nasr P et al (2015) Fibrosis stage is the strongest predictor for disease-specific mortality in NAFLD after up to 33 years of follow-up. Hepatology 61(5):1547-1554. https://doi. org/10.1002/hep.27368

37. Wu WC, Wang CY (2013) Association between non-alcoholic fatty pancreatic disease (NAFPD) and the metabolic syndrome: casecontrol retrospective study. Cardiovasc Diabetol 12:77. https://doi. org/10.1186/1475-2840-12-77

38. Patel AV, Rodriguez C, Bernstein L et al (2005) Obesity, recreational physical activity, and risk of pancreatic cancer in a large U.S. cohort. Cancer Epidemiol Biomark Prev 14(2):459-466. https:// doi.org/10.1158/1055-9965.epi-04-0583

39. Yamashita H, Takenoshita M, Sakurai M et al (2001) A glucoseresponsive transcription factor that regulates carbohydrate metabolism in the liver. Proc Natl Acad Sci U S A 98(16):9116-9121. https://doi.org/10.1073/pnas.161284298

40. Ferre P, Foufelle F (2010) Hepatic steatosis: a role for de novo lipogenesis and the transcription factor SREBP-1c. Diabetes Obes Metab 12(Suppl. 2):83-92. https://doi.org/10.1111/j.1463-1326. 2010.01275.x

41. Lavine JE, Schwimmer JB, Van Natta ML et al (2011) Effect of vitamin $\mathrm{E}$ or metformin for treatment of nonalcoholic fatty liver disease in children and adolescents: the TONIC randomized controlled trial. JAMA 305(16):1659-1668. https://doi.org/10. 1001/jama.2011.520

Publisher's note Springer Nature remains neutral with regard to jurisdictional claims in published maps and institutional affiliations. 


\section{Affiliations}

Mohammad S. Kuchay ${ }^{1}$ - Sonal Krishan ${ }^{2}$ - Sunil K. Mishra ${ }^{1}$ - Narendra S. Choudhary ${ }^{3} \cdot$ Manish K. Singh $^{4}$. Jasjeet S. Wasir ${ }^{1} \cdot$ Parjeet Kaur ${ }^{1} \cdot$ Harmandeep K. Gill $^{1} \cdot$ Tarannum Bano $^{1} \cdot$ Khalid J. Farooqui $^{1} \cdot$ Ambrish Mithal $^{1}$

1 Division of Endocrinology and Diabetes, Medanta-The Medicity Hospital, Haryana, India

2 Department of Radiology, Medanta-The Medicity Hospital, Haryana, India
3 Institute of Digestive and Hepatobiliary Sciences, Medanta-The Medicity Hospital, Haryana, India

4 Department of Clinical Research and Studies, Medanta-The Medicity Hospital, Haryana, India 